\title{
Multiplet Effects in the Quasiparticle Band Structure of the $f^{1}-f^{2}$ Anderson Model
}

\author{
B.R. Trees \\ Department of Physics, Monmouth College, Monmouth, IL 61462 \\ A.J. Fedro \\ Physics Department, Northern Illinois University, DeKalb, IL 60115 \\ and Materials Science Division, Argonne National Laboratory, Argonne, IL 60439 \\ M.R. Norman \\ Materials Science Division, Argonne National Laboratory, Argonne, IL 60439
}

(December 15, 2018)

\begin{abstract}
In this paper, we examine the mean field electronic structure of the $f^{1}-$ $f^{2}$ Anderson lattice model in a slave boson approximation, which should be useful in understanding the physics of correlated metals with more than one $f$ electron per site such as uranium-based heavy fermion superconductors. We find that the multiplet structure of the $f^{2}$ ion acts to quench the crystal field splitting in the quasiparticle electronic structure. This is consistent with experimental observations in such metals as $U P t_{3}$.
\end{abstract}

71.27.+a, 71.28.+d

Typeset using REVTEX 
Localized electron systems exhibit multiplets of definite L, S, and J due to the symmetry of the Coulomb interaction about an atomic site. The degeneracies of these multiplets are not properly described by standard electronic structure techniques used in solids which assume the filling of single particle levels using Fermi-Dirac statistics. Despite this, a Landau quasiparticle approach appears to be successful even in metals which exhibit high energy multiplet excitations. A good example of this is the heavy fermion superconductor $\mathrm{UPt}_{3}$. This metal exhibits canonical Fermi liquid behavior at low temperatures with a Fermi surface in reasonable agreement with band calculations 1 . On the other hand, high energy neutron scattering data reveal an excitation at about $0.4 \mathrm{eV}$, free ion, known to be a multiplet excitation between the ${ }^{3} H_{4}$ ground state and the ${ }^{3} F_{2}$ excited state. Therefore, $\mathrm{UPt}_{3}$ is quite unusual in that its low energy excitations exhibit single particle Kramers degeneracy whereas its high energy ones exhibit multi-particle Coulomb degeneracy. Our purpose here is to understand what influence these $f^{2}$ multiplet correlations have on the low energy quasiparticle excitations. We do this by studying the $f^{1}-f^{2}$ Anderson lattice model at the mean field level within a slave boson approximation. Since this study deals with the relation of single particle statistics to multi-particle statistics, it should be of relevance to other problems as well.

The $f^{1}-f^{2}$ Anderson model has been investigated by a number of authors. Early work studied the single impurity model either from a variational approach 3 a or by use of the $1 / \mathrm{N}$ expansion 5 where $\mathrm{N}$ is the degeneracy of the ion. The lattice case was in turn looked at by various authors within a slave boson approximation, 6 , but the effects of multiplets were ignored. Multiplet notation was introduced in what we will denote the $m n$ basis by Newns and Read, 1 l but no calculations were carried out explicitly including multiplet effects (the $m n$ state has one $\mathrm{f}$ electron in level $\mathrm{m}$ and the other in level $\mathrm{n}$ ). This work was extended by Dorin and Schlottmann 10 who dealt with the general $f^{n}-f^{n+1}$ problem in the $m n$ basis and also included Gutzwiller corrections to the mean field approximation, which are necessary when looking for possible magnetic instabilities 11 Our treatment in the $m n$ basis will closely parallel this work. In turn, though, we will develop the formalism in the $J J_{z}$ basis, where $\mathrm{J}$ is the total angular momentum of the $f^{2}$ state. This basis is diagonal in the Coulomb interaction and contains the proper multiplet degeneracy of the $f^{2}$ ion.

The ionic part of the Hamiltonian in the $m n$ basis is

$$
\sum_{j m} E_{m}|m><m|+\sum_{j m n} E_{m n}|m n><m n|
$$

where $E_{m}$ is the energy of one $\mathrm{f}$ electron in level $\mathrm{m}, E_{m n}$ is the energy of two $\mathrm{f}$ electrons in levels $\mathrm{m}$ and $\mathrm{n}$, and $\mathrm{j}$ is the site index. Note that the state $\mid m n>$ is antisymmetric in the two indices. We will now assume that we are in the strong spin-orbit coupling limit (as appropriate for uranium) so that the $f^{1}$ state has a $\mathrm{J}$ of $5 / 2$ (6 states) and the $f^{2}$ state has a $\mathrm{J}$ of 0,2 , or 4 ( 15 states).

The conduction electron part of the Hamiltonian is

$$
\sum_{\vec{k} m} \epsilon_{\vec{k} m} c_{\vec{k} m}^{\dagger} c_{\vec{k} m}
$$

where $\mathrm{m}$ is assumed to run over the same 6 indices as the $f^{1}$ state.

The hybridization part of the Hamiltonian is 


$$
\sum_{j \vec{k} m n} V_{\vec{k} m} e^{i \vec{k} \cdot \vec{R}_{j}}|m n><n| c_{\vec{k} m}+H . C .
$$

where H.C. denotes the Hermitian conjugate. In this paper we replace $V_{\vec{k} m}$ by a constant, $V$.

To solve this Hamiltonian, we employ the slave boson approximation. We associate a boson $p_{j m}$ at each site $\mathrm{j}$ for each $f^{1}$ state in level $\mathrm{m}$ and a boson $d_{j m n}$ at each site $\mathrm{j}$ for each $f^{2}$ state in level $\mathrm{mn}$. For the mean field approximation we consider, these bosons are replaced by c numbers assumed to be site independent. The ionic part of the Hamiltonian per site can now be written as

$$
\sum_{m} E_{m} p_{m}^{2}+\sum_{m n} E_{m n} d_{m n}^{2}
$$

We must also satisfy a completeness relation

$$
\sum_{m} p_{m}^{2}+\sum_{m n} d_{m n}^{2}=1
$$

We associate a Lagrange multiplier $\lambda^{1}$ with this constraint. The other constraints involve the $\mathrm{f}$ count in each channel $m$ at each site

$$
h_{m} \equiv f_{m}^{\dagger} f_{m}=p_{m}^{2}+\sum_{n} d_{m n}^{2}
$$

with which we associate Lagrange multipliers $\lambda_{m}$.

Finally, the hybridization term now becomes

$$
\sum_{\vec{k} m} V f_{\vec{k} m}^{\dagger} c_{\vec{k} m} z_{m}+H . C .
$$

where

$$
z_{m}=\sum_{n} d_{m n} p_{n}
$$

and $f_{\vec{k} m}$ is the Fourier transform of $f_{j m}$. The Gutzwiller generalization would involve replacing $z_{m}$ by

$$
\tilde{z}_{m}=\frac{z_{m}}{\sqrt{\left(1-h_{m}\right) h_{m}}}
$$

We do not use such a renormalization here, but it can be easily included.

We now have to determine the following parameters: $6 p_{m}, 15 d_{m n}, \lambda^{1}$, and $6 \lambda_{m}$. These are determined by setting the derivative of the Hamiltonian with respect to each of them equal to zero. The final parameter is the chemical potential, $\mu$, determined by the total number of electrons.

The quasiparticle bands which come out of this model are the standard ones

$$
E_{\vec{k} m}^{ \pm}=\frac{\lambda_{m}+\epsilon_{\vec{k} m}}{2} \pm \frac{1}{2} \sqrt{\left(\lambda_{m}-\epsilon_{\vec{k} m}\right)^{2}+4 z_{m}^{2} V^{2}}
$$


The following mean field relations are necessary to solve these equations (we assume a constant conduction electron density of states, $\left.N_{0}\right)^{8}$

$$
\begin{gathered}
\sum_{\vec{k}}<c_{\vec{k} m}^{\dagger} c_{\vec{k} m}>=N_{0}\left(\mu-\epsilon_{a}^{m}\right) \\
\sum_{\vec{k}}<f_{\vec{k} m}^{\dagger} f_{\vec{k} m}>=N_{0} z_{m}^{2} V^{2}\left(\frac{1}{\epsilon_{a}^{m}-\lambda_{m}}-\frac{1}{\mu-\lambda_{m}}\right) \\
\sum_{\vec{k}} R e<f_{\vec{k} m}^{\dagger} c_{\vec{k} m}>=N_{0} z_{m} V \ln \left(\frac{\mu-\lambda_{m}}{\epsilon_{a}^{m}-\lambda_{m}}\right)
\end{gathered}
$$

which we denote by $I_{m}, J_{m}$, and $K_{m}$ respectively where $\epsilon_{a}^{m}$ is the bottom of the $E_{m}^{-}$band. These equations as written assume only the $E_{m}^{-}$bands are occupied for notational simplicity, but in actual calculations, we used the full expressions which can include contributions from the $E_{m}^{+}$bands depending on the value of the chemical potential, $\mu$. We note that the f count, $n_{f}$, is simply the sum over $m$ of the $J_{m}$.

The $m n$ basis is not diagonal in the Coulomb interaction, so to treat the effect of multiplets properly, we convert the above formalism to the $J J_{z}$ basis. We note that

$$
\left|J J_{z}>=\sum_{m n} U_{J J_{z}}^{m n}\right| m n>
$$

where the $U$ are Clebsch-Gordon coefficients. 2 These act to define a new boson field for the $f^{2}$ state which we denote as $d_{J J_{z}}$.

The ionic part of the Hamiltonian per site is now

$$
H_{\text {ionic }}=\sum_{m} E_{m} p_{m}^{2}+\sum_{J J_{z}} E_{J J_{z}} d_{J J_{z}}^{2}
$$

with the completeness relation

$$
\sum_{m} p_{m}^{2}+\sum_{J J_{z}} d_{J J_{z}}^{2}=1
$$

To convert Eqs. 6 and 8, though, one must remember that $d$ is actually an operator and that $d_{m n}$ and $d_{J_{z}}$ represents its expectation value in each basis. Thus, taking advantage of the assumed diagonality in the $J J_{z}$ basis, we have

$$
\begin{aligned}
& d_{m n}=\sum_{J J_{z}} U_{J J_{z}}^{m n} d_{J J_{z}} U_{J J_{z}}^{m n} \\
& d_{m n}^{2}=\sum_{J J_{z}} U_{J J_{z}}^{m n} d_{J J_{z}}^{2} U_{J J_{z}}^{m n}
\end{aligned}
$$

These two equations are then inserted into Eqs. 6 and 8 to obtain the values of $h_{m}$ and $z_{m}$ in the $J J_{z}$ basis. 
Solving the full problem above is quite difficult given the large number of variational parameters. We will therefore consider a simple model which illustrates the relevant points. In this model, we only keep one $f^{2}$ configuration, the doublet $J, J_{z}=4, \pm 4$. Since the $f^{1}$ state $\mid \pm 1 / 2>$ does not couple to this, we elect to keep only the $\mid \pm 3 / 2>$ and $\mid \pm 5 / 2>$ states in the $f^{1}$ and conduction electron manifolds, thus further reducing the number of variational parameters. Since we are considering an unpolarized solution, one has only the following equations to solve

$$
\begin{aligned}
2\left(p_{3 / 2}^{2}+p_{5 / 2}^{2}+d_{44}^{2}\right)-1 & =0 \\
p_{5 / 2}^{2}+d_{44}^{2}-J_{5 / 2} & =0 \\
p_{3 / 2}^{2}+d_{44}^{2}-J_{3 / 2} & =0 \\
\left(E_{44}+\lambda^{1}-\lambda_{5 / 2}-\lambda_{3 / 2}\right) & d_{44} \\
+V\left(K_{5 / 2} p_{3 / 2}+K_{3 / 2} p_{5 / 2}\right) & =0 \\
\left(E_{5 / 2}+\lambda^{1}-\lambda_{5 / 2}\right) p_{5 / 2}+V K_{3 / 2} d_{44} & =0 \\
\left(E_{3 / 2}+\lambda^{1}-\lambda_{3 / 2}\right) p_{3 / 2}+V K_{5 / 2} d_{44} & =0 \\
2\left(J_{3 / 2}+J_{5 / 2}+I_{3 / 2}+I_{5 / 2}\right)-N_{t} & =0
\end{aligned}
$$

with $z_{5 / 2}=d_{44} p_{3 / 2}, z_{3 / 2}=d_{44} p_{5 / 2}$, and $N_{t}$ the total number of electrons. We solve these seven equations by squaring them all and adding them together. The resulting master equation is a function of seven variables and is minimized using Powell's method.13

For the results presented, we took a conduction band width of $2 \mathrm{eV}$, a hybridization potential, $V$, of $0.7 \mathrm{eV}$, an $E_{5 / 2}$ of $-0.5 \mathrm{eV}$, an $E_{3 / 2}$ of $-0.4 \mathrm{eV}$, and a total electron count of 3.5. $E_{44}$ was varied in steps of $0.05 \mathrm{eV}$. Stable solutions were found for $E_{44} 0.13 \mathrm{eV}$ and lower. For higher values of $E_{44}$, the minimiziation failed to converge from a typical input. A solution at $0.15 \mathrm{eV}$ was generated by trying a large number of input parameters. For 0.20 $\mathrm{eV}$, the lowest minimum was found for an $n_{f}$ of 1 . For low values of $E_{44}$, one asymptotically approaches an $n_{f}$ of 2 , so the runs were terminated at $-1.50 \mathrm{eV}$.

The results are presented in the three figures. Fig. 1 shows a plot of the renormalized crystal field splitting, $\Delta \lambda \equiv \lambda_{3 / 2}-\lambda_{5 / 2}$, versus $n_{f}$. The data for $n_{f}>1.2$ appears to follow a straight line which goes to zero at $n_{f}=2$ and interpolates to half the bare crystal field splitting at $n_{f}=1$. Below an $n_{f}$ of 1.2 , the chemical potential jumps from the lower to the upper $5 / 2$ band. After this, the crystal field splitting begins to approach the unrenormalized value of $0.1 \mathrm{eV}$ (the lowest minimum for $E_{44}$ of $0.20 \mathrm{eV}$ had an $n_{f}$ of 1 and an unrenormalized crystal field splitting). We contrast this behavior with the $f^{0}-f^{1}$ Anderson lattice model, where no renormalization of the crystal field splitting occurs. In Fig. 2, a plot of $\lambda_{5 / 2}-\mu$ is shown, which should be an estimate of the Kondo temperature for this model (the absolute value is shown, since this quantity changes sign near an $n_{f}$ of 1.2 ). This vanishes linearly with $n_{f}$ as $n_{f}$ approaches 2 but vanishes faster than this when $n_{f}$ approaches 1 , with the latter behavior beginning when $n_{f}$ drops below 1.2. This same type of asymmetry was seen in earlier impurity calculations. $\mathrm{E}$ In Fig. 3, we show the f occupation in each channel as a function of $n_{f}$. Again, a change in behavior occurs when $n_{f}$ drops below 1.2 with very different limiting behavior as $n_{f}$ approaches 1 or 2 . We suggest, then, that the jump of the chemical potential is necessary for the solution to cross over from the behavior characteristic of $n_{f}$ near 2 to obtain the unrenormalized behavior as $n_{f}$ approaches 1 . The value of $n_{f}$ at which this occurs is model dependent. 
We now present a derivation of the linear behavior of Fig. 1. Subtracting Eq. 24 from Eq. 23 and utilizing the defintion of $K_{m}$ in Eq. 13, we have

$$
\Delta \lambda=\Delta \lambda_{0}+N_{0} V^{2} d_{44}^{2} \ln \left[\frac{\lambda_{3 / 2}-\mu}{\lambda_{5 / 2}-\mu} \frac{\epsilon_{a}^{5 / 2}-\lambda_{5 / 2}}{\epsilon_{a}^{3 / 2}-\lambda_{3 / 2}}\right]
$$

where $\Delta \lambda=\lambda_{5 / 2}-\lambda_{3 / 2}$ and $\Delta \lambda_{0}=E_{5 / 2}-E_{3 / 2}$. As $n_{f}$ approaches 2 , we can set the second fraction in the argument of the logarithm to 1 , and expanding the logarithm, we obtain

$$
\Delta \lambda \simeq \Delta \lambda_{0}-N_{0} V^{2} d_{44}^{2} \frac{\Delta \lambda}{\lambda_{a v}-\mu}
$$

where $\lambda_{a v}=\frac{1}{2}\left(\lambda_{5 / 2}+\lambda_{3 / 2}\right)$. This approximation has been checked numerically and works well for $n_{f}$ near 2 . To get an expression for $\lambda_{a v}-\mu$, we analyze the definition of $J_{m}$ in Eq. 12. Since $z_{m}$ is vanishing as $n_{f}$ approaches 2 , this forces $\lambda_{m}$ to approach $\mu$, and so we can ignore the first term in parenthesis in Eq. 12. Using Eqs. 20 and 21, we obtain

$$
\lambda_{a v}-\mu \simeq N_{0} V^{2} d_{44}^{2} \frac{p_{a v}^{2}}{p_{a v}^{2}+d_{44}^{2}}
$$

where $p_{a v}^{2}=\frac{1}{2}\left(p_{5 / 2}^{2}+p_{3 / 2}^{2}\right)$. From Eqs. 19-21, $4 p_{a v}^{2}=2-n_{f}$, so

$$
\lambda_{a v}-\mu \simeq N_{0} V^{2} d_{44}^{2} \frac{2-n_{f}}{n_{f}}
$$

Substituting this into Eq. 27, we obtain

$$
\Delta \lambda \simeq \frac{\Delta \lambda_{0}}{2}\left(2-n_{f}\right)
$$

which is the desired relationship.

Fig. 1 is the main result of this paper. We note that except for the special cases of the $J=4, J_{z}= \pm 4, \pm 3$ states, all $f^{2}$ states involve combinations of states in all three of the $f^{1}$ subbands. Because of this, in general, all crystal field splittings in the quasiparticle electronic structure should be expected to vanish in the limit as $n_{f}$ approaches 2 in the paramagnetic case. In fact, this effect should occur in the Kondo limit for any $f^{n}-f^{n+1}$ problem with $n \neq 0$ (the $n=0$ case is special since the $f^{0}$ state has zero $\mathrm{f}$ electrons). We suggest this as a possible reason for the lack of any definite evidence for crystal field splittings in $U P t_{3}$. Evidence for crystal field effects have been seen in other heavy fermion superconductors $\left(U R u_{2} S i_{2}, U P d_{2} A l_{3}\right)$, but these are a reflection of the crystal field splitting of the $f^{2}$ manifold, not of the quasiparticles states. We note that in these cases, the ground state is magnetic, causing a profound effect on the quasiparticle electronic structure, so the above considerations based on a paramagnetic solution would not apply (a weak moment state is found in $U P t_{3}$, but it does not appear to affect the quasiparticle electronic structure).

In conclusion, by studying the $f^{1}-f^{2}$ Anderson lattice model, we find that multi-particle correlations have a profound impact on the quasiparticle electronic structure. The results of this paper should have particular bearing on uranium heavy fermion metals where such multi-particle ground states are known to exist. 


\section{ACKNOWLEDGMENTS}

This work was supported by the U.S. Dept. of Energy, Basic Energy Sciences, under Contract No. W-31-109-ENG-38. 


\section{REFERENCES}

${ }^{1}$ L. Taillefer and G.G. Lonzarich, Phys. Rev. Lett. 60, 1570 (1988).

${ }^{2}$ R. Osborn, K.A. McEwen, E.A. Goremychkin, A.D. Taylor, Physica B 163, 37 (1990).

${ }^{3}$ Y. Yafet, C.M. Varma, and B. Jones, Phys. Rev. B 32, 360 (1985).

${ }^{4}$ A.C. Nunes, J.W. Rasul, and G.A. Gehring, J. Phys. C 19, 1017 (1986).

${ }^{5}$ N. Read, K. Dharamvir, J.W. Rasul, and D.M. Newns, J. Phys. C 19, 1597 (1986).

${ }^{6}$ J.W. Rasul and A.P. Harrington, J. Phys. C 20, 4783 (1987).

${ }^{7}$ S.M.M. Evans and G.A. Gehring, J. Phys. Cond. Mat. 1, 10487 (1989).

${ }^{8}$ D.M. Newns and N. Read, Adv. Phys. 36, 799 (1987).

${ }^{9}$ D.M. Newns, Phys. Rev. B 36, 2429 (1987).

${ }^{10}$ V. Dorin and P. Schlottmann, Phys. Rev. B 47, 5095 (1993).

${ }^{11}$ G. Kotliar and A.E. Ruckenstein, Phys. Rev. Lett 57, 1362 (1986).

${ }^{12}$ E.U. Condon and H. Odabasi, Atomic Structure (Cambridge Univ. Pr., Cambridge, 1980), p. 145.

${ }^{13}$ W.H. Press, B.P. Flannery, S.A. Teukolsky, and W.T. Vetterling, Numerical Recipes (Cambridge Univ. Pr., Cambridge, 1989), Section 10.5. 


\section{FIGURES}

FIG. 1. Renormalized crystal field splitting, $\Delta \lambda$, versus $n_{f}$, with a bare splitting of $0.1 \mathrm{eV}$. The straight line is an analytic approximation valid near an $n_{f}$ of 2 derived in the text. The change in behavior near an $n_{f}$ of 1.2 is due to a jump in the chemical potential from the lower to the upper $5 / 2$ quasiparticle band.

FIG. 2. Absolute value of the separation of the renormalized $\mathrm{f}$ level position in the $5 / 2$ channel from the chemical potential versus $n_{f}$, which should be an estimate of the Kondo temperature.

FIG. 3. f electron counts in the $3 / 2$ and $5 / 2$ channels versus $n_{f}$. 


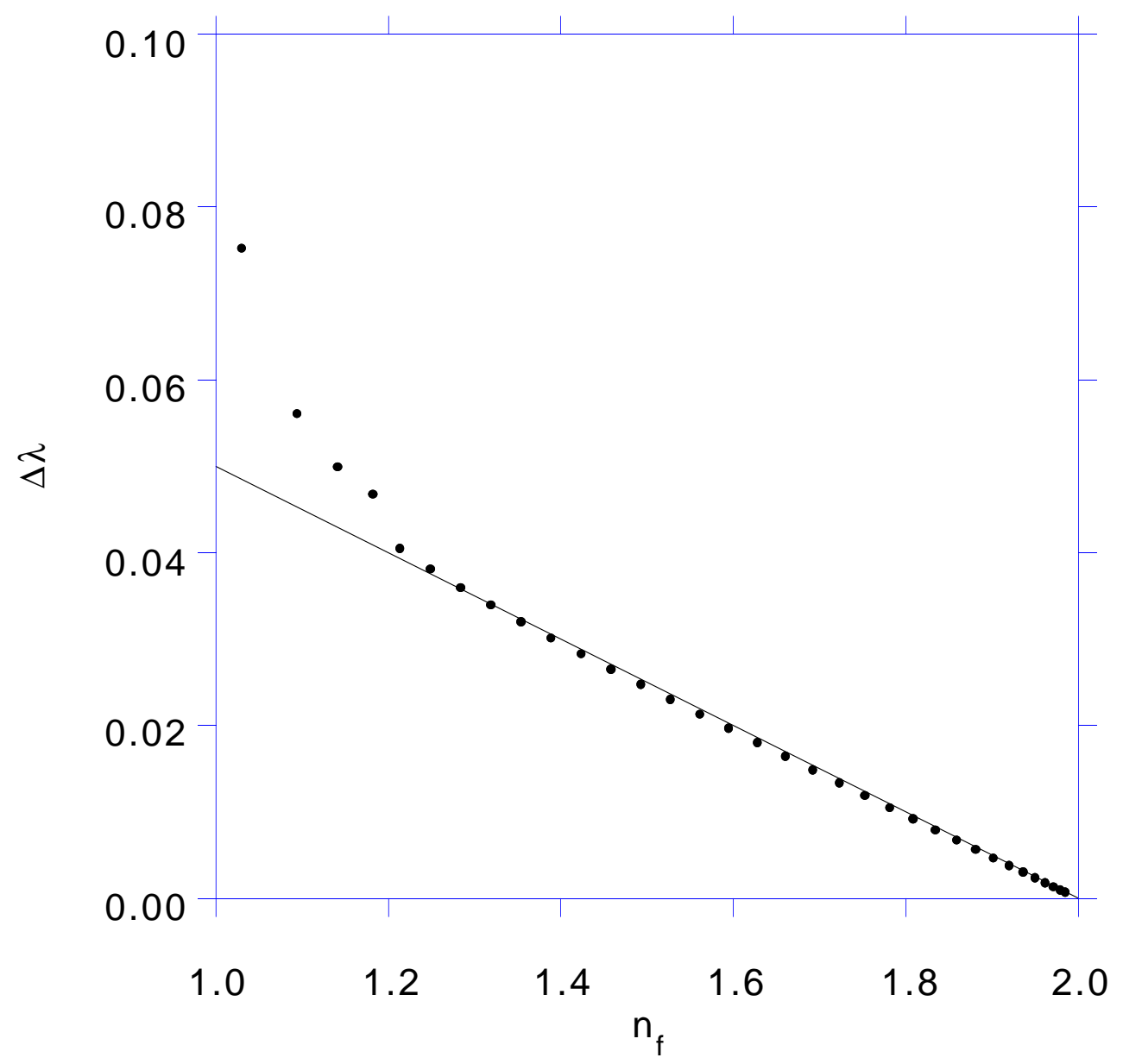




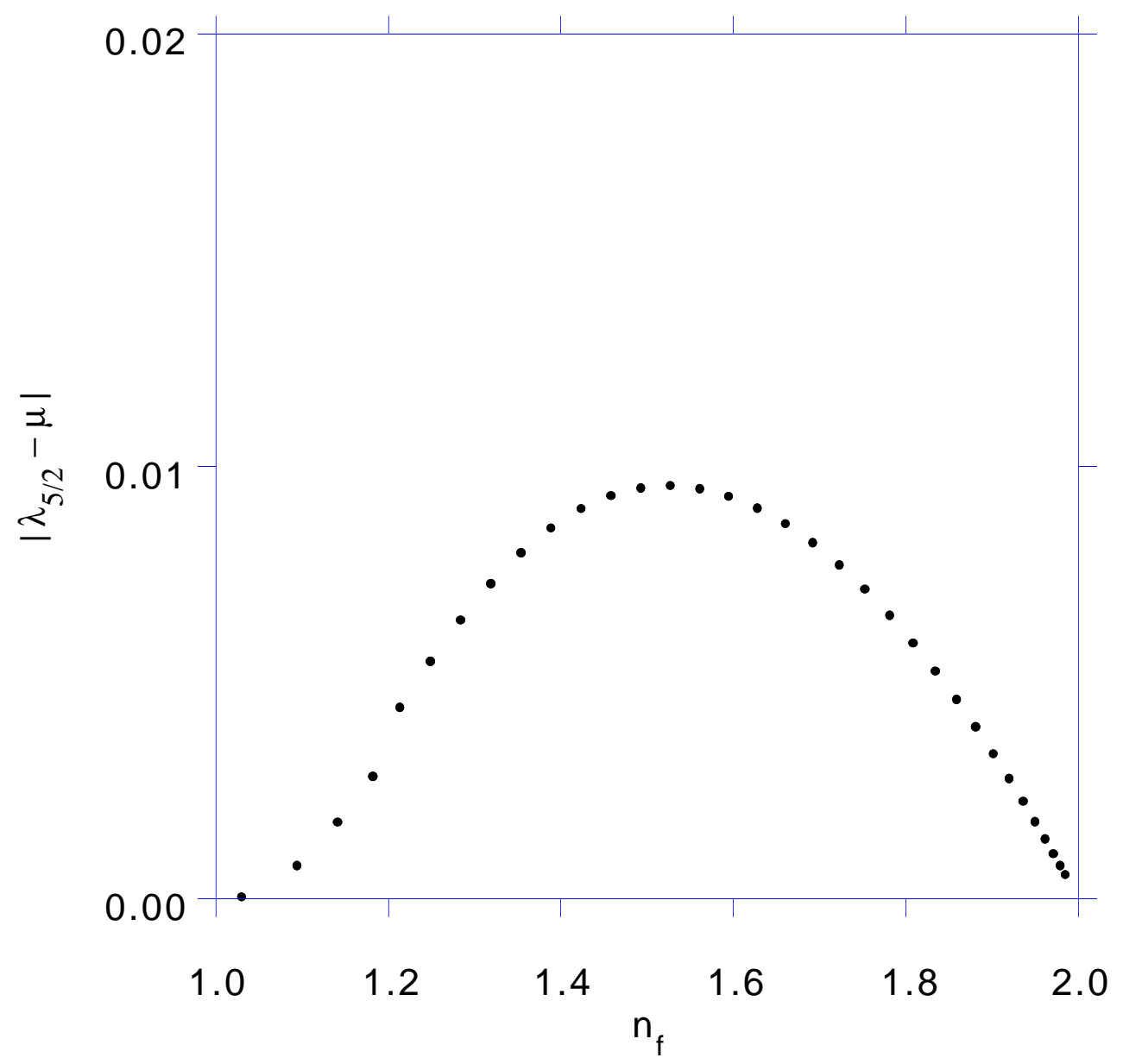




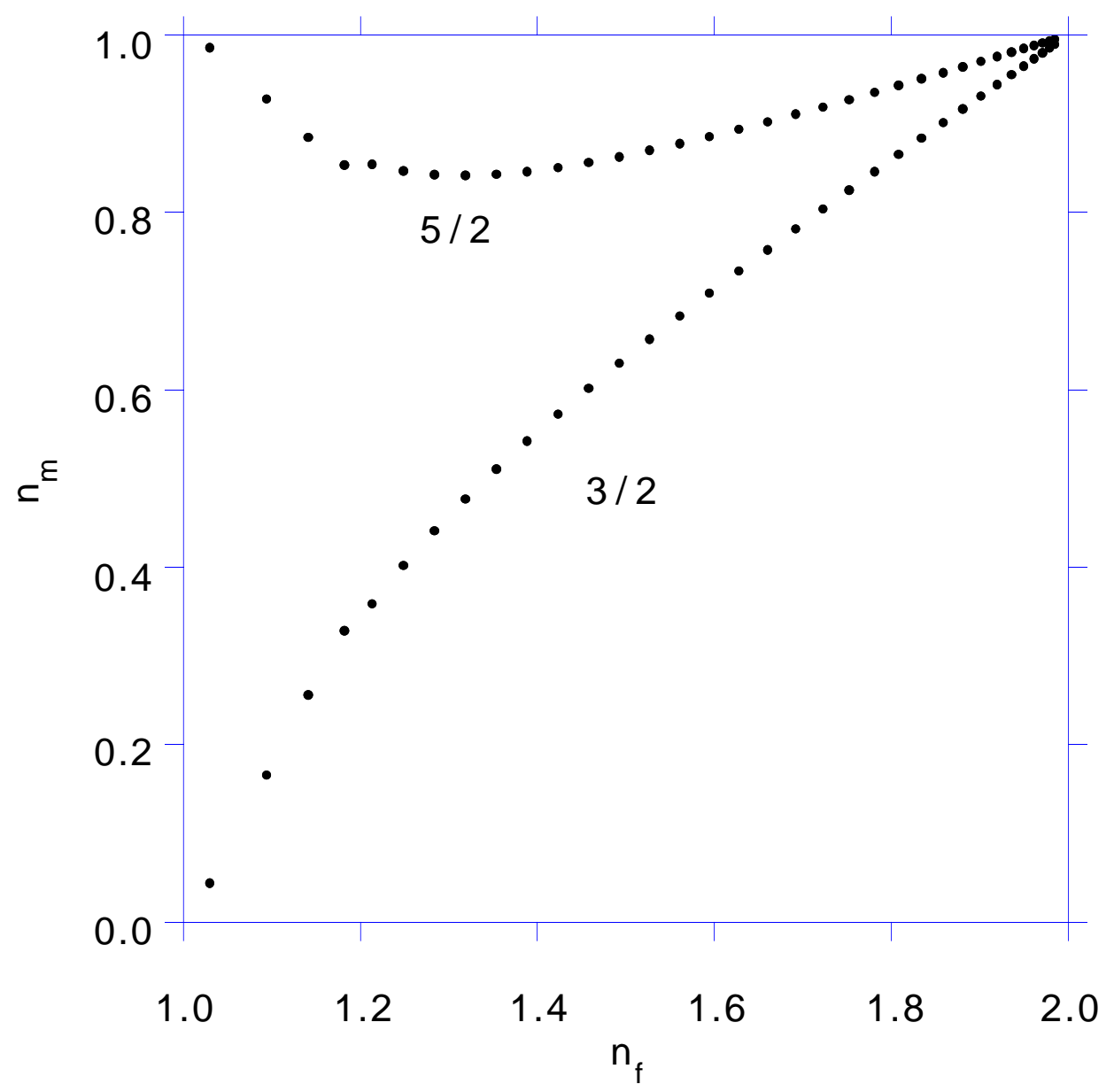

